\title{
Frontières
}

\section{Déficience intellectuelle et résilience}

\section{Yves Boisvert}

Volume 22, numéro 1-2, automne-printemps 2009-2010

\section{Résilience et deuil}

URI : https://id.erudit.org/iderudit/045034ar

DOI : https://doi.org/10.7202/045034ar

Aller au sommaire du numéro

Éditeur(s)

Université du Québec à Montréal

ISSN

1180-3479 (imprimé)

1916-0976 (numérique)

Découvrir la revue

\section{Citer cet article}

Boisvert, Y. (2009). Déficience intellectuelle et résilience. Frontières, 22(1-2), 99-107. https://doi.org/10.7202/045034ar

\section{Résumé de l'article}

Vouloir situer le concept de résilience dans la philosophie d'intervention en " déficience intellectuelle " peut apparaître, à première vue, un objectif assez inusité, car nous reconnaissons peu à ces personnes les capacités nécessaires pour relever le défi que représente leur intégration ou leur participation sociale. Concevoir la résilience comme un processus au lieu d'une capacité permet de considérer ce concept dans le réseau de la déficience intellectuelle à travers trois sens particuliers : historique, théorique et clinique. Le recours à une adaptation clinique du Processus de production du handicap (PPH) favorise une compréhension au quotidien des interactions entre les facteurs de risque et les facteurs de protection qui placent la personne présentant des incapacités intellectuelles en situation de vulnérabilité ou de résilience.
Ce document est protégé par la loi sur le droit d'auteur. L'utilisation des services d'Érudit (y compris la reproduction) est assujettie à sa politique d'utilisation que vous pouvez consulter en ligne.

https://apropos.erudit.org/fr/usagers/politique-dutilisation/ 


\section{Résumé}

Vouloir situer le concept de résilience dans la philosophie d'intervention en "déficience intellectuelle» peut apparaître, à première vue, un objectif assez inusité, car nous reconnaissons peu à ces personnes les capacités nécessaires pour relever le défi que représente leur intégration ou leur participation sociale. Concevoir la résilience comme un processus au lieu d'une capacité permet de considérer ce concept dans le réseau de la déficience intellectuelle à travers trois sens particuliers: historique, théorique et clinique. Le recours à une adaptation clinique du Processus de production du handicap (PPH) favorise une compréhension au quotidien des interactions entre les facteurs de risque et les facteurs de protection qui placent la personne présentant des incapacités intellectuelles en situation de vulnérabilité ou de résilience.

Mots clés: résilience - vulnérabilité déficience intellectuelle - Processus de production du handicap - intervention.

\section{Abstract}

Trying to position the resilience concept in a mental retardation intervention philosophy can seem at first an odd objective because we often do not recognize to these individuals the capacity to face the challenges of their integration or social participation. Seeing resilience as a process instead of an ability allows this concept in the mental retardation network through three particular meanings: historical, theoretical and clinical. The clinical adaptation of the Disability Creation Process model helps a daily understanding of the interactions between the risks and the protection factors that put the person with mental disabilities in a situation vulnerability or resilience.

Keywords: resilience - vulnerability intellectual deficiency - Disability

Creation Process - intervention.

$\begin{array}{lllllllllllll}\text { I } & N & T & E & R & V & E & N & T & \text { I } & O & N\end{array}$

\title{
DÉFICIENCE INTELLECTUELLE ET RÉSILIENCE
}

\begin{abstract}
SI JE PARVIENS À CHANGER VOTRE REGARD SUR MOI, JE CHANGERAIS LE SENTIMENT QUE J'ÉPROUVE DE MOI.
\end{abstract}

BORIS CYRULNIK, UN MERVEILLEUX MALHEUR, P. 14.

Yves Boisvert, M.A. en psychoéducation, agent de programmation, de planification et de recherche, CRDI Normand-Laramée (Laval, Québec).

\section{« BONJOUR, JE M'APPELLE JEAN, J'AI UNE DÉFICIENCE INTELLECTUELLE ET JE SUIS RÉSILIENT. »}

Cette phrase, simple en apparence, énonce trois affirmations dont l'une souligne un fait véridique, une autre, une imprécision et la dernière, un étonnement. Autrement dit, ce titre fait état de ce qui a été chèrement acquis au fil des ans par les familles et les mouvements associatifs concernés par la «déficience intellectuelle», de ce qui a été dépassé par l'avancement des connaissances et du changement de vision des intervenants et des professionnels qui œuvrent dans le domaine de la déficience intellectuelle et de ce qui est grandement espéré par les personnes elles-mêmes qui présentent une "déficience intellectuelle».

Placer côte à côte les notions de "résilience» et de «déficience intellectuelle» peut paraître déconcertant pour plusieurs, non pas à cause du sens accordé au mot "résilience», mais bien à cause de la croyance de ne pas trouver chez la personne qui présente une «déficience intellectuelle» les aptitudes qu'un tel concept requiert pour reconnaître sa présence.

Exprimé de cette façon, le concept de résilience semble se réduire à un fait singulier, celui de posséder les capacités permettant de faire face à une situation d'adversité. Comme jadis au sujet du handicap ou de la déficience, ce serait, une fois de plus, situer les facteurs déterminants à l'intérieur de la personne. Aborder les aspects liés à la philosophie d'intervention dans le domaine de la déficience intellectuelle requiert une vision plus actuelle de ce que recouvre la notion de «déficience intellectuelle», du jeu interactif des facteurs en cause, mais surtout de l'importance accordée au contexte de vie dans lequel la personne cherche à s'intégrer.

Dans cette perspective, lorsqu'il est question de "réadaptation", il ne s'agit plus uniquement de bien saisir les problèmes vécus par les personnes ou les familles, mais de les aborder «autrement», 
dans un effort de compréhension de ce que peut signifier le développement de l'empowerment, du pouvoir d'agir des personnes et des familles, en tenant compte, cette fois-ci, de leurs croyances, de leurs capacités d'exécution et de décision, ainsi que de la mobilisation des ressources humaines et techniques concernées par leur situation. De plus, vouloir composer avec la résilience nous amène à considérer nos actions dans une dynamique évolutive qui nécessite de nombreux ajustements, tant de la part des personnes que de celle des milieux dans lesquels elles évoluent.

La résilience peut difficilement être abordée sans s'attarder à donner un sens à nos actions dans le cadre d'une relation d'accompagnement des personnes et des familles. Dans un premier temps, nous dégagerons un "sens historique » à cette relation, en situant la place occupée par la résilience dans la pratique professionnelle en déficience intellectuelle, mais aussi dans les mouvements sociaux fondateurs de notre histoire. Puis, un «sens théorique» sera précisé en intégrant le concept de résilience dans une adaptation clinique du processus de production $\mathrm{du}$ handicap en déficience intellectuelle. En tout dernier lieu, nous nous appliquerons à définir un «sens clinique » en orientant nos réflexions sur l'élaboration d'une philosophie d'intervention qui tracera les grands principes à considérer lorsque nous tenons à faire de la résilience, un concept central de notre pratique professionnelle.

Souvenez-vous de cette phrase clé : «Bonjour, je m'appelle Jean, j'ai une déficience intellectuelle et je suis résilient.» Elle sera le fil conducteur de ce texte.

\section{UN SENS HISTORIQUE}

De la fin des années 1950 jusqu'au début des années 1970, l'approche médicale faisait de la personne un "déficient mental» porteur de son handicap. L'intervention privilégiée consistait à corriger les comportements du "déficient» par des méthodes comportementalistes auxquelles nous ajoutions une prise de médicaments. L'évolution de la pratique professionnelle dans le domaine de la déficience intellectuelle se résume en grande partie par le passage d'un modèle médical à un modèle écologique pour expliquer le fonctionnement d'une personne. Ce passage aura pris plus de quarante années et nécessité la contribution de quatre grands mouvements sociaux redevables, en très grande partie, aux actions des familles et des mouvements de défense des droits de la personne. Ces mouvements, dont les racines sont d'origine scandinave, portent le nom de normalisation, de valorisation des rôles sociaux, d'intégration sociale et de participation sociale (Boisvert, à paraître $)^{1}$. Depuis que nous connaissons le concept de résilience, nous serions tentés de définir ces mouvements sociaux comme relevant de divers processus de résilience familiale, communautaire et environnementale.

Si l'utilisation du mot "résilience» est passablement récente dans le champ de la déficience intellectuelle, et que son influence sur la pratique professionnelle est passablement mitigée, la réalité qu'il recouvre se marie très bien avec l'organisation des services et la transformation progressive des pratiques professionnelles. Avant même de connaître ce que recouvre ce concept, des expressions comme facteurs de risque, facteurs de protection, vulnérabilité, contexte de vie, projet personnel, incapacités et situation de handicap étaient utilisées pour décrire la réalité vécue par une personne ayant une déficience intellectuelle. Le fait de considérer la résilience comme le résultat d'une interaction entre les facteurs de risque et les facteurs de protection n'est pas étranger à cette affirmation.

L'historique des services en déficience intellectuelle mène à la reconnaissance de l'individu en tant que personne. Effectivement, l'un des gains les plus significatifs a été celui de ne plus réduire l'individu à l'une de ses caractéristiques, la déficience intellectuelle, et de le considérer d'abord comme une personne: «Je m'appelle Jean. » Pour demeurer fidèle à la résilience, il nous reste à faire en sorte que cette personne ait sa propre histoire.

La philosophie d'accompagnement développée au fil des ans place la personne et sa famille au cœur de toute la démarche évaluative. Le point de départ de tout accompagnement réside dans la définition, par la personne elle-même ou de son représentant (si elle est trop jeune ou si elle présente des incapacités trop importantes), d'un projet éducatif ou personnel à l'actualisation duquel l'intervenant contribuera. Contribuer à l'actualisation d'un projet nous apparaît plus prometteur que de chercher à répondre à des besoins qui, par définition, sont sans cesse renouvelés.

La centration sur la réalisation d'un projet a pour principale conséquence de faire glisser nos actions sur l'interaction entre la personne et son environnement. Et c'est cette dynamique interactive qui fonde les concepts de «participation sociale» et de «résilience». Ces concepts n'ont de sens que par leur association à la notion de contexte (Anaut, 2006; Terrisse et Lefebvre, 2007). La trajectoire de vie des personnes est formée par la juxtaposition de l'ensemble de ces contextes où les facteurs environnementaux donnent la répartie aux facteurs personnels pour forger, à la personne et à sa famille, une histoire de vie conséquente et la plus harmonieuse possible.

La résilience personnelle apparaît grandement tributaire des effets de la résilience familiale et de la disponibilité des ressources de l'environnement physique et social pouvant assurer un soutien technique et humain auprès des familles. Dans ce contexte comme dans bien d'autres, nous constatons l'impact prépondérant des facteurs environnementaux sur les facteurs génétiques. La plasticité cérébrale, les effets de la rémédiation cognitive et l'influence des facteurs épigénétiques, pour ne nommer que ceux-ci, permettent d'installer une vision d'espoir là où le déterminisme semble avoir tissé sa toile.

Une même altération chromosomique mènera à un important déficit intel-

lectuel dans un milieu affectif appauvri alors que les performances seront bien meilleures dans un milieu sécurisant et stimulant (Plomin cité par Cyrulnik) [...] Malgré la forte détermination génétique, il est donc tout de même possible de mettre en place un processus de résilience (Cyrulnik, 2001, p. 137).

Mais ce processus de résilience, comment se met-il en place?

\section{UN SENS THÉORIQUE}

La philosophie d'accompagnement qui guide les actions dans le soutien apporté aux personnes qui présentent une déficience intellectuelle a connu une lente progression qui s'est soldée par le passage du modèle médical au modèle écologique. Le fait d'avoir dégagé un sens historique a permis d'introduire la notion de résilience en la juxtaposant à celles de participation sociale et d'empowerment. Puis, la différenciation progressive des mouvements sociaux qui ont marqué les quarante dernières années dans le domaine de la déficience intellectuelle situe tous les efforts d'adaptation et d'intégration des personnes dans une dynamique interactionnelle.

Jean a désormais une existence qui lui est propre, mais celle-ci demeure grandement tributaire de l'aide que peuvent lui apporter sa famille, les organismes de défense des droits de la personne et les différents intervenants du réseau de services. La section historique du présent texte se termine sur une note positive mais quelque peu fragile et incertaine. Il est possible, nous dit Boris Cyrulnik (2001), de mettre en place un processus de résilience chez les personnes qui ont vécu une situation traumatique, de même que chez celles dont le bagage génétique a connu une altération chromosomique qui a mené à un important 
déficit cognitif. Mais à quoi pourrait ressembler le processus de résilience s'il était appliqué à l'ensemble des personnes qui présentent une déficience intellectuelle? Le Processus de production du handicap (PPH) (Fougeyrollas et al., 1998) permet d'analyser et de comprendre le rôle des facteurs en jeu lorsqu'une personne se trouve en situation de handicap. Intégrons à ce modèle conceptuel, les notions de résilience, les facteurs de protection et de vulnérabilité qui interagissent tout au long de la trajectoire de vie d'une personne.

\section{UN APERÇU DU PROCESSUS DE PRODUCTION DU HANDICAP (PPH)}

Le Processus de production du handicap (PPH) est, d'abord et avant tout, un modèle anthropologique du développement humain, élaboré par une équipe de professionnels québécois œuvrant dans le domaine de la déficience physique. Il s'agit d'une contribution originale qui, en s'inscrivant dans la foulée des travaux de l'Organisation mondiale de la santé dans le cadre de la révision de la Classification internationale des déficiences, des incapacités et des handicaps, a réussi à faire considérer le handicap comme étant le résultat situationnel d'un processus

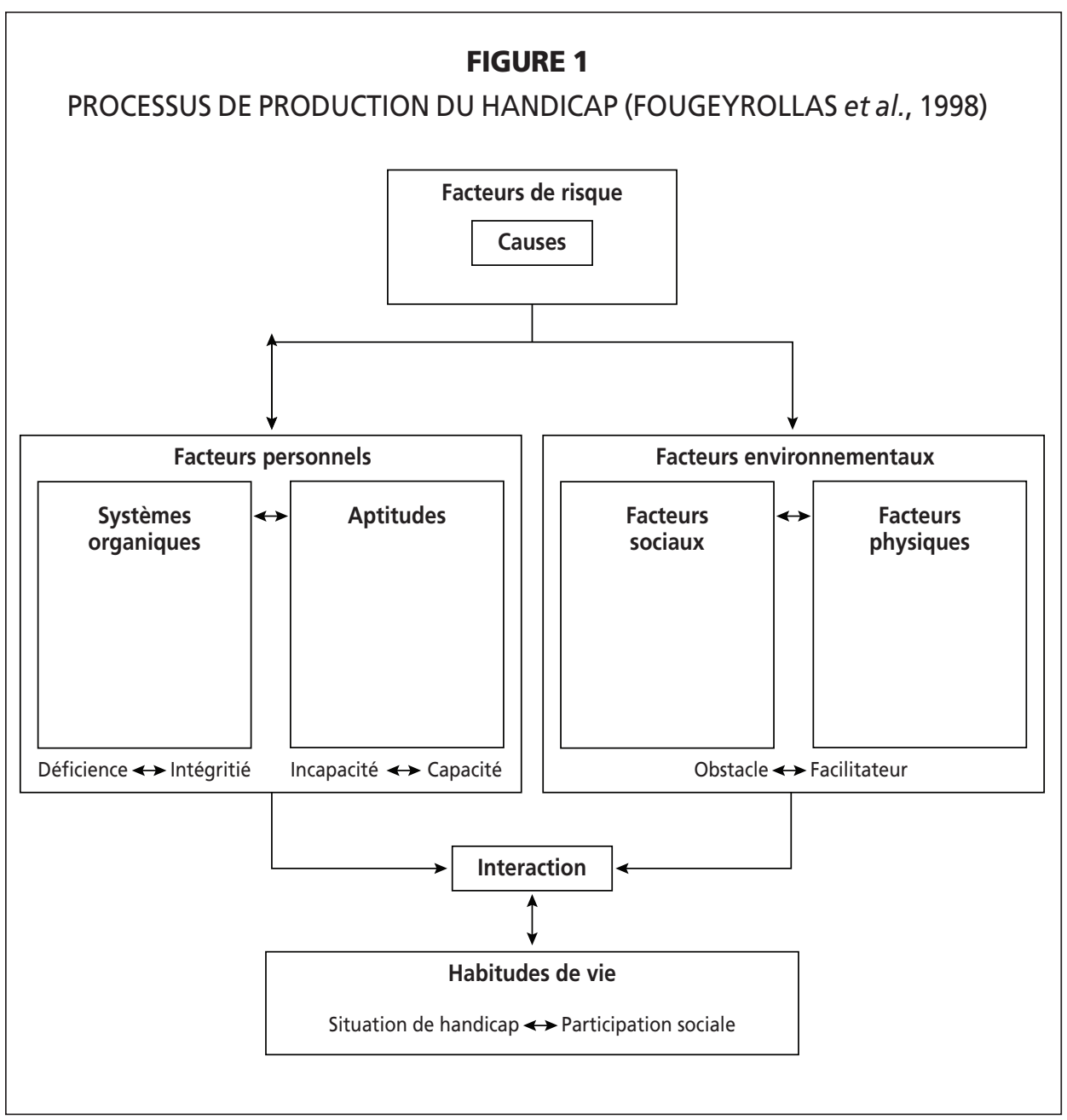

interactif entre les caractéristiques de la personne et les caractéristiques de l'environnement. Ce faisant, le handicap quittait le domaine purement subjectif pour occuper un espace mitoyen entre la personne et ses environnements.

Le modèle du $\mathrm{PPH}$ possède une grande valeur heuristique, car il est aussi un modèle d'analyse des causes et conséquences des maladies, traumatismes et autres atteintes à l'intégrité ou au développement de la personne. De ce seul fait, il laisse envisager de nombreux liens avec le concept de résilience, sans pour autant les identifier de manière explicite. Dans le modèle $\mathrm{PPH}$, un facteur de risque (traumatisme, maladie, accident, syndrome génétique, anomalie chromosomique, etc.) peut entraîner une déficience des systèmes organiques qui aura des répercussions sur les aptitudes physiques et mentales de la personne. Si cette dernière ne reçoit pas de soutien de la part de sa famille ou de son milieu social, et si des obstacles physiques se dressent devant elle, l'interaction de tous ces facteurs fera en sorte qu'au niveau des habitudes de vie (éducation, loisir, nutrition, déplacements, travail, etc.), elle se trouvera en situation de handicap (figure 1). 
lisation du modèle conceptuel du PPH, une personne présentera une déficience du système nerveux central qui entraînera chez elle l'apparition d'un certain nombre d'incapacités intellectuelles. À moins de se référer au diagnostic lui-même dont l'appellation de «retard mental» demeure inchangée (pour l'instant), lorsque sont décrites les caractéristiques cognitives d'une personne, cette dernière présente des incapacités intellectuelles et non plus une déficience intellectuelle. La personne n'est plus globalement «déficiente», et le fait de présenter des incapacités intellectuelles rend nécessaire leur identification. Cette façon de nommer les choses indique que peuvent cohabiter chez la personne des limitations mais aussi des forces en termes de capacités. L'American Association on Mental Retardation (AAMR) (2002), avant de devenir l'AAIDD en 2006, souligne cette coexistence dans l'un des postulats essentiels à l'utilisation de sa définition du retard mental: "Chez une même personne, les limitations coexistent souvent avec des forces. » Avant d'aborder le concept de résilience, il nous apparaît important de mettre en évidence l'existence de forces chez les personnes qui présentent des incapacités intellectuelles. Il restera à préciser si ces forces sont suffisantes pour permettre à la personne de se considérer comme étant une personne résiliente.

Souvenez-vous encore: "Bonjour, je m'appelle Jean, j'ai une déficience intellectuelle et je suis résilient. » À la lumière des précisions que nous venons d'apporter, cette phrase devrait se lire ainsi: «Bonjour, je m'appelle Jean, je suis une personne qui présente des incapacités intellectuelles et...».

Qu'en est-il au juste de la dernière affirmation: «Je suis résilient»?

\section{UNE ADAPTATION CLINIQUE DU PPH EN DÉFICIENCE INTELLECTUELLE}

Le modèle conceptuel du PPH permet aux intervenants qui œuvrent dans le domaine de la déficience intellectuelle de mieux saisir la genèse d'une situation de handicap par le jeu interactif des facteurs personnels et des facteurs environnementaux. Par contre, même si le PPH permet d'analyser les facteurs contributifs à une situation de handicap, parce qu'il a pour objectif de déterminer le degré actuel des désavantages et des limitations vécus par la personne, il ne met l'accent que sur la contribution d'événements présents ou de ceux associés au passé récent d'une personne et de sa famille. Dans le domaine de la déficience intellectuelle, les intervenants demeurent centrés sur la situation présente mais ils essaient aussi de dégager la trajectoire de vie d'une personne et de sa famille. La raison en est bien simple: les principaux facteurs contributifs à une situation de handicap exercent leur influence bien souvent depuis la naissance de la personne. Les limitations constatées chez une personne ne sont que très rarement le fruit d'une situation causée par un événement unique (accident ou maladie) et récent qui agirait comme facteur déterminant. L'analyse de la situation en cause doit se baser sur l'identification d'une série d'événements qui ont tous contribué (dans une mesure différente) à entraîner une ou plusieurs situations de handicap. Dans le PPH, ces événements sont considérés comme étant des facteurs de risque dont certains, par leur action déterminante sur la situation, s'appellent des causes.

Mais il existe aussi dans le cas des personnes présentant une déficience intellectuelle (comme pour celles ayant une déficience physique ou autres), des événements ou des personnes qui ont joué un rôle important dans leur vie, en essayant de contrecarrer les effets négatifs des facteurs de risque, notamment la présence d'adultes bienveillants, de parents compétents qui ont donné à leur enfant une bonne éducation, un milieu familial véhiculant des valeurs cohérentes, un professeur attentif à développer chez la personne une bonne estime d'elle-même, des activités valorisantes, des co-travailleurs qui permettent à la personne de donner un sens à son travail, etc. Ces facteurs qui exercent des effets positifs en favorisant le développement d'une personnalité saine, stable et unifiée, sont appelés des facteurs de protection.

Dans l'application clinique du PPH en déficience intellectuelle, l'existence des facteurs de risque et des facteurs de protection permet de considérer une première dynamique interactive qui favorisera, selon la qualité des facteurs en présence, l'apparition d'un contexte de vulnérabilité ou d'un contexte de résilience. Cette intuition de départ a trouvé la confirmation dans la plupart des ouvrages consultés sur la résilience. Il existe un consensus important à ce niveau. Le fait d'avoir inscrit ces deux catégories de facteurs dans une perspective temporelle au niveau de la trajectoire de vie d'une personne exprime très bien le choix de considérer la résilience comme étant, en premier lieu, un processus qui peut s'établir tout au long de la vie et, en second lieu, une capacité lorsque nous la considérons en fonction de son influence concrète dans une situation donnée (voir figure 2).

Pour bien saisir cette dynamique interactive qui se joue entre les facteurs de risque et les facteurs de protection, il est important d'approfondir quelque peu ces notions. Pour situer la place de la résilience dans l'intervention en déficience intellectuelle, nos explications se centreront uniquement sur la première boucle interactive de notre application clinique. Cette section du modèle représente les interactions entre les facteurs de risque et les facteurs de protection qui ont influencé la trajectoire de vie de la personne et dont certains conservent une influence sur la situation de handicap vécue par la personne.

Le principal objectif fixé concerne le développement des capacités de la personne qui présente des incapacités intellectuelles. Pour ce faire, nous devons prendre en considération les facteurs biologiques, psychodéveloppementaux et socio-environnementaux dont les interactions pourraient avoir entraîné, soit des déficiences au plan des systèmes organiques, soit des incapacités au plan des aptitudes, et qui, de ce fait, ont rendu cette personne plus vulnérable à toute situation de stress ou d'adversité, ou avoir favorisé un développement plus harmonieux en termes d'intégrité et de capacités, et rendu ainsi cette personne plus apte à mettre à contribution sa capacité de résilience. Pour des auteurs comme Misès, Perron et Salbreux (1994), rien ne peut être construit sur le plan de l'accompagnement tant que l'étape de la recherche étiologique n'a pas été réalisée et les résultats transmis à la personne et aux familles.

\section{LES FACTEURS DE RISQUE}

Un facteur de risque est un élément appartenant à l'individu ou provenant de l'environnement susceptible de contribuer à provoquer une maladie, un traumatisme ou toute autre atteinte à l'intégrité ou au développement de l'organisme. Parmi les grandes catégories des facteurs de risque, le RIPPH (Fougeyrollas et al., 1998) propose les quatre catégories suivantes: les risques biologiques (ex.: les anomalies chromosomiques), les risques liés à l'environnement physique (ex.: les produits chimiques), les risques liés à l'organisation sociale (ex. : la pauvreté) ou ceux liés aux comportements individuels et sociaux (ex. : l'hyperactivité).

L'influence de ces facteurs de risque ne doit pas être analysée d'une manière isolée. Le caractère multifactoriel du processus étiologique est reconnu par la grande majorité des professionnels de sorte que nous ne pouvons plus catégoriser les causes selon leur origine biologique ou psychosociale. Les facteurs de risque sont multiples et c'est la convergence de leurs effets qui prédispose un individu à vivre des situations de handicap ou de participation sociale. Concernant la clientèle cible, il est rapporté qu'environ 50\% des 


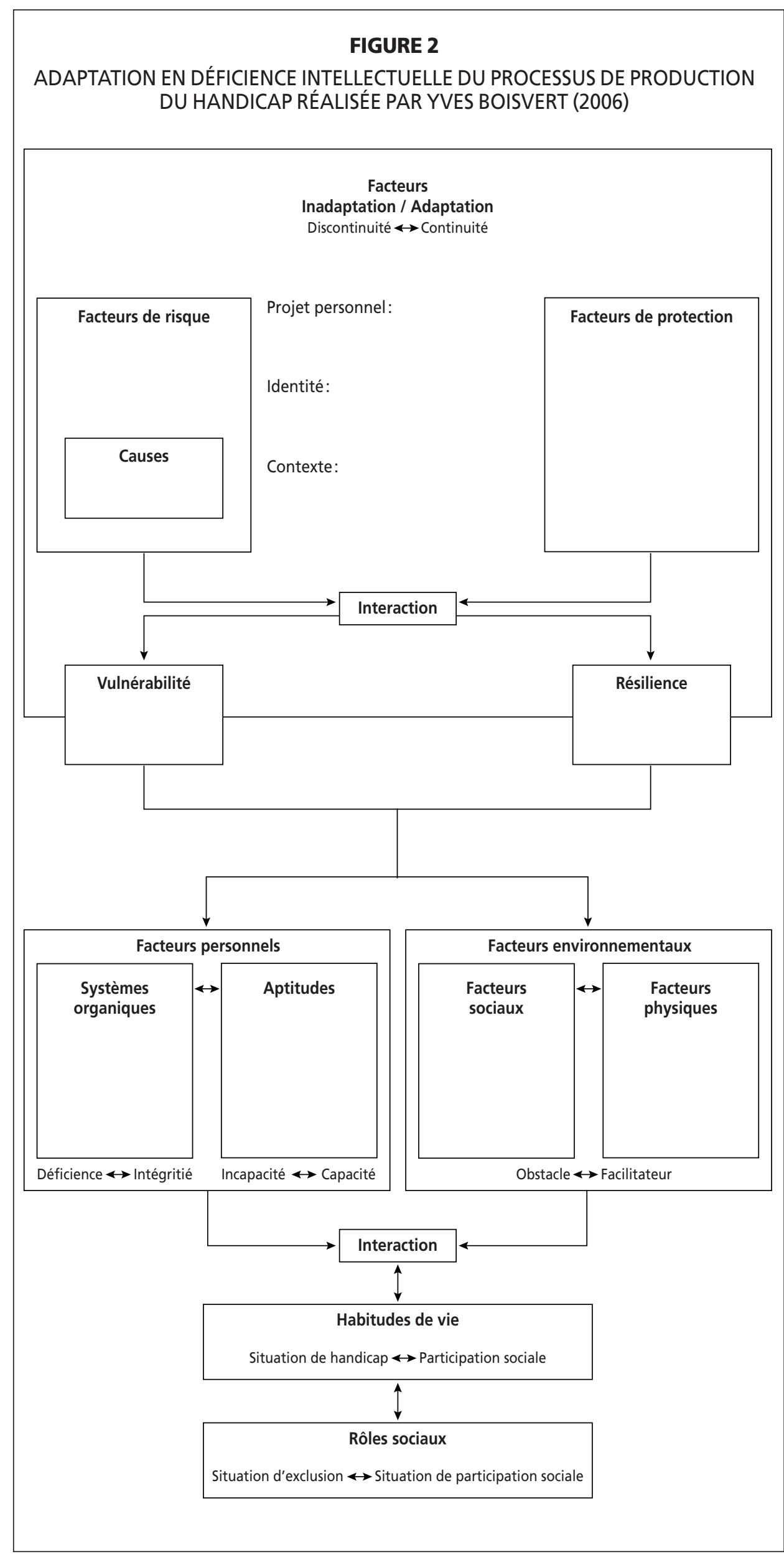

individus présentant une déficience intellectuelle ont souvent plus d'un facteur de risque expliquant le handicap. De plus, la déficience intellectuelle résulte souvent des effets cumulatifs ou interactifs des facteurs de risque.

Établir l'étiologie de la déficience intellectuelle permet de comprendre les processus impliqués qui sont les déterminants du fonctionnement intellectuel de la personne. La recherche des facteurs causals aide à émettre des hypothèses sur la genèse d'une situation de handicap, de manière à ce qu'une formulation efficace de ces facteurs contienne déjà des germes de solutions (Tremblay, 1992).

Dans ce sens, il est intéressant de constater qu'il existe des parcours de développement différents selon que la déficience intellectuelle est associée à une origine biologique où nous retrouvons des personnes avec des quotients intellectuels (QI) plus faibles ou qu'elle est associée à une origine d'ordre psychosocial où nous retrouvons une majorité de personnes dans la catégorie des déficiences intellectuelles légères.

\section{LA VULNÉRABILITÉ}

Il nous faut préciser avec Theis (2001) que les personnes sont inégales devant le risque: elles sont plus ou moins vulnérables. Selon cet auteur, la notion de vulnérabilité implique que l'on prenne en compte: les probabilités génétiques, les différences constitutionnelles, les hasards de la reproduction, les maladies physiques, les traumatismes dus à l'environnement, les situations d'adversité et les crises développementales. Dans les écrits scientifiques sur la question, le continuum vulnérabilitéinvulnérabilité a été abandonné au profit du continuum vulnérabilité-résilience, car si le premier continuum permettait de mettre en évidence les difficultés d'adaptation et les problèmes comportementaux chez des personnes ayant connu des événements qui ont exercé une influence négative sur leur développement, il ne permettait pas de comprendre pourquoi parmi les personnes «à haut risque » certaines ne succombaient pas à la pathologie. De plus, Rutter et Rutter (1993) précisent que des personnes invincibles, ça n'existe pas; certains individus sont plus résistants que d'autres, mais tout le monde a ses limites. Il souligne aussi un point essentiel: le concept d'invulnérabilité semble correspondre à une caractéristique intrinsèque à l'individu alors que les recherches montrent que son origine est à situer dans le contexte social, comme nous le verrons pour le concept de résilience.

Si nous avons ajouté au modèle conceptuel du PPH la partie positive manquante ${ }^{2}$ que sont les facteurs de protection, c'est 
aussi à cause de l'idée de Rutter et Rutter qui recommandent de s'intéresser aux mécanismes de risque et non pas aux facteurs de risque, car la même caractéristique peut être un risque dans une situation et un facteur de protection dans une autre.

Ainsi proposée, cette référence aux mécanismes de risque indique que c'est l'exposition au risque qui provoque la mise en jeu des mécanismes de résilience (Michaud, 2003), et que c'est la maîtrise de la situation de risque qui favorise le développement des potentialités de la personne (Petit et al., 1999). Au cours de notre pratique professionnelle auprès des personnes présentant des incapacités intellectuelles, nous avons mainte fois constaté que les effets des facteurs de risque peuvent être cumulatifs. Un enfant qui présente, dès la naissance, un retard important dans plusieurs sphères de son développement, et qui se retrouve dans un milieu familial qui accentue cette vulnérabilité à cause d'une situation financière précaire qui entraîne des conditions de vie où la privation et la maltraitance sont habituelles, éprouvera beaucoup plus de difficulté à se développer harmonieusement et à s'intégrer dans ses milieux de vie qu'un autre enfant qui aurait connu une trajectoire de vie marquée par un nombre moins grand de facteurs de risque. Bernardini et Jenkins (2002) donnent une description des effets cumulatifs du risque sur le développement des enfants. Les effets des facteurs de protection peuvent cependant réduire le degré de vulnérabilité présent chez ces enfants.

Selon la philosophie d'intervention adoptée, il est pertinent de préciser que les interventions concernent le développement optimal d'une personne en visant la création d'un équilibre entre le pouvoir d'agir de la personne et le pouvoir d'agir de sa famille et de son réseau d'entraide. Notre accompagnement et nos interventions ont pour objectif de restaurer cet équilibre, soit en diminuant l'exposition à des facteurs de risque ou à des événements stressants, soit en rendant plus efficients les facteurs de protection individuels, familiaux ou environnementaux.

En résumé, il est reconnu que:

- la génétique peut exercer une influence sur l'aptitude cognitive;

- la contribution biologique à l'intelligence ne reste pas la même au cours de la vie;

- les conditions environnementales peuvent améliorer ou entraver le développement et le bien-être de la personne;

- l'état de santé physique peut affecter le fonctionnement général;
- les diverses composantes cognitives contribuent distinctement aux efforts d'adaptation et d'intégration de la personne dans ses divers milieux de vie.

Les facteurs de risque sont des facteurs personnels ou environnementaux (sociaux et physiques) qui, considérés sur une base temporelle longitudinale, contribuent à former avec les facteurs de protection, la trajectoire de vie de la personne. Il est par contre fort difficile d'évaluer l'interaction entre ces facteurs. Ce qu'il nous faut retenir, c'est que l'expérience a un effet considérable sur les capacités cognitives de la personne.

\section{LES FACTEURS DE PROTECTION}

Comme nous l'avons décrit ci-dessus, nous avons associé aux facteurs de risque une catégorie de facteurs qui leur sont théoriquement opposés. Il s'agit des facteurs de protection. Rutter et Rutter (1993) définissent les facteurs de protection de la façon suivante: ce sont des caractéristiques, des conditions ou des circonstances qui diminuent l'impact des facteurs de risque sur la personne ou favorisent la «résistance» de celle-ci aux facteurs de risque.

À titre d'exemples de facteurs de protection, mentionnons: l'estime de soi, la sociabilité, le don d'éveiller la sympathie, un certain sens de l'humour, un projet de vie, un quotient intellectuel élevé, une bonne capacité de résolution de problèmes, une capacité de planification, un sentiment d'efficacité personnelle, etc. En observant ces facteurs de plus près, il apparaît assez clairement que la grande majorité des personnes qui présentent des incapacités intellectuelles en sont grandement démunies.

C'est la présence des facteurs de protection qui permet de considérer les aspects positifs de la dynamique interactive entre les facteurs personnels et les facteurs environnementaux. Dans cette dynamique particulière, les facteurs de protection n'existent que par leur relation avec les facteurs de risque, sinon ils sont considérés comme étant des facteurs de développement. C'est par leurs effets sur les facteurs de risque qu'ils rendent possible le développement de la résilience.

Pouvons-nous accorder aux facteurs de protection, une des propriétés que nous avons reconnues aux facteurs de risque: l'effet cumulatif? Il existe, en effet, une propriété semblable qui porte plus d'un nom mais qui est souvent mentionnée à partir de son mode d'élaboration. À la suite de Cyrulnik, plusieurs auteurs font mention dans leurs écrits sur la résilience de la métaphore du «tissage de liens». Cette dernière prend en compte l'interaction entre les éléments qui proviennent de la réalité personnelle et ceux qui proviennent du contexte de vie. La mécanique n'est pas la même que celle du risque, car les effets des facteurs de protection ne sont décelables qu'en réaction à une situation d'adversité. Le tissage de liens entre la personne et ses environnements s'effectue tout au long de la vie de la personne de sorte que l'effet réel d'un facteur ne peut s'exercer que plusieurs années plus tard. Selon la qualité des liens qui ont été tissés, la personne pourra faire face, dans diverses modalités, à une situation d'adversité. Un bébé qui sourit reçoit plus de réactions positives qui contribuent au développement de l'estime de lui-même, qui, à son tour, contribuera à son succès scolaire qui lui apportera de nouvelles reconnaissances, et ainsi de suite.

Dans notre adaptation clinique du modèle $\mathrm{PPH}$, les facteurs de protection se retrouvent au niveau de la trajectoire de vie des personnes, dans la première boucle interactive. De ce fait, pour être reconnu à titre de facteur de protection, un élément positif doit démontrer un effet réel sur un facteur de risque qui persiste dans le temps. Cette nuance est importante, car elle permet d'apporter une distinction entre les aptitudes (facteurs personnels), les facilitateurs (facteurs environnementaux) et les facteurs de protection. À titre d'exemple, si dans notre analyse d'une situation de handicap, nous constatons qu'une personne démontre une certaine confiance en soi malgré le fait qu'elle vient de perdre son emploi ou qu'elle vient d'être victime d'un accident, cette aptitude sera considérée comme facteur de protection seulement si elle représente une manière constante de réagir face à ce type de situation d'adversité. Sinon, la confiance en soi restera collée au contexte en cause et correspondra à une aptitude.

Cette relativité temporelle et contextuelle des facteurs de protection comme des facteurs de risque nous amène à définir le «processus de résilience».

\section{LA RÉSILIENCE}

Le résilience est définie comme étant le résultat de l'interaction entre les facteurs de risque et les facteurs de protection (Anaut, 2007; Terrisse et al., 2007; Lecompte, 2004).

La résilience est une réalité qui existe depuis toujours et qui se manifeste à partir d'une situation difficile qui peut prendre des formes très variées :

- un traumatisme ponctuel et brutal comme le décès d'une personne proche; - un traumatisme répété comme des abus sexuels ou physiques;

- une déficience physique permanente comme une paraplégie; 
- une situation à haut risque comme celle d'un enfant vivant dans un milieu défavorisé où règne la négligence;

- une maladie grave comme le Parkinson ou une autre maladie dégénérative;

- ou des situations fréquentes d'adversité comme la perte d'un emploi, les déménagements, l'absence d'un parent significatif, etc.

Lorsque nous prenons en considération l'ensemble de ces facteurs, il est permis d'ajouter à cette liste, le fait de venir au monde avec des incapacités intellectuelles, car si nous tenons compte des facteurs étiologiques et environnementaux en cause, cette situation engendre des conséquences aussi dramatiques pour les personnes elles-mêmes que pour leur entourage immédiat.

Il existe plusieurs définitions de la résilience mais elles s'apparentent grandement à celle proposée par Vanistendael (1998, p. 8), sauf en ce qui concerne la précision sur le développement socialement acceptable: "La résilience est la capacité à réussir, à vivre et à se développer positivement, de manière socialement acceptable, en dépit du stress ou d'une adversité qui comportent normalement le risque grave d'une issue négative. »

La résilience n'est jamais absolue ou totale, ni acquise une fois pour toutes, et elle est variable selon les circonstances. Pour des personnes vivant en contexte d'adversité, être résilient peut aussi impliquer des comportements de survie qui ne correspondent pas à des conduites en accord avec les normes sociales. Par contre, à chaque instant, la résilience résulte de l'interaction entre l'individu lui-même et son entourage, entre les empreintes de son vécu antérieur et le contexte du moment en matière politique, économique, sociale et humaine (Manciaux et al., 2001).

Revenons à la troisième affirmation de la personne présentant une déficience intellectuelle: «Je suis résilient. » La résilience étant un processus, elle ne peut s'appliquer à représenter une caractéristique ou une capacité globale d'une personne. Dans le domaine de la déficience intellectuelle, les intervenants sont sensibles à cet aspect, car pendant de nombreuses décennies (et encore aujourd'hui mais d'une façon moindre), les personnes qui présentent une déficience intellectuelle ont fait l'objet d'une stigmatisation en voyant leur identité réduite à cette seule caractéristique cognitive. Il faut éviter une fois de plus ce piège, en voulant faire des personnes, des résilients. Balegno et Colmaneres (2003, p. 156) expriment très bien cette impasse, en parlant des enfants des rues en Colombie:

Confondre la situation de vie de ces [enfants] avec leur identité les enferme dans une catégorie qui rend difficile la possibilité d'un redémarrage. Par contre, penser ces [enfants] en termes de résilience permet de mettre en lumière des aptitudes et des capacités vitales qui devraient pouvoir faire partie des ressources à considérer dans un projet d'aide.

LE CONCEPT DE RÉSILIENCE PERMET DE MIEUX ÉVALUER OU DE MIEUX NOMMER LE RÉSULTAT DE L'INTERACTION ENTRE LES FACTEURS DE RISQUE ET LES FACTEURS DE PROTECTION. IL PERMET ESSENTIELLEMENT DE CRÉER UN CONTINUUM «SITUATION DE VULNÉRABILITÉ ET SITUATION DE RÉSILIENCE », QUI NOUS AIDE À MIEUX DÉCRIRE LES CONTEXTES DE VIE DE LA PERSONNE.

La résilience n'est pas acquise une fois pour toutes et une personne peut faire preuve de résilience dans un contexte comme le travail, mais se retrouver en situation de handicap dans son milieu familial. Ainsi, résilience et situation de handicap procèdent toutes les deux d'une même dynamique interactive, la première au niveau de la trajectoire de vie et la seconde, au niveau des habitudes de vie.

En tenant compte de ces distinctions, l'expression «personne résiliente» est inappropriée, car la résilience se vit en contexte. Une personne peut manifester des comportements de résilience, mais elle ne peut être globalement une personne résiliente.

Compte tenu de l'analyse effectuée, notre phrase clé peut être modifiée de la façon suivante: "Bonjour, je m'appelle Jean, je suis une personne qui présente des incapacités intellectuelles et qui peut manifester certains comportements résilients. »

Le développement de la résilience est, en quelque sorte, un nouveau regard porté sur la réalité de la personne en vue d'un meilleur usage des stratégies d'intervention. Au-delà des symptômes et des comportements, il est primordial de chercher à détecter et à mobiliser les ressources des personnes et de leur entourage, ainsi que celles des services et des réseaux sociaux, éducatifs et sanitaires. Ce regard s'apparente très bien à celui que pose le RIPPH sur la réalité des personnes lorsqu'il insère dans son modèle conceptuel, la dimension positive que représentent les notions d'intégrité, de capacité, de facilitateurs et de participation sociale.
Défini à l'origine en fonction de l'individu, le concept de résilience s'insère de plus en plus dans une conception systémique ou une écologie sociale qui procède par une analyse de l'interaction entre les divers systèmes qui entourent la personne. Pour un auteur comme Garmezy (1993), les écrits sur la résilience reconnaissent de plus en plus le rôle de l'environnement, de la famille et du soutien social dans leurs 
Fombonne fait bien ressortir cette dynamique surtout lorsqu'il rapporte le point de vue suivant: "Le défi ne consiste plus désormais à identifier de nouveaux facteurs de risque ou de protection, mais à élucider les processus à l'œuvre, en particulier dans l'interaction entre les facteurs génétiques et l'environnement» (cité par Bouvier, 2001, p. 53).

Si le processus de résilience se développe au fil de la trajectoire de vie, il se mesure par contre au temps présent. Le sens clinique accordé au processus de résilience prend sa pleine mesure lorsqu'est défini son rôle au quotidien. L'adaptation clinique du PPH que nous avons réalisée permet de dégager la contribution de l'ensemble des facteurs sur le fait qu'une personne se trouve en situation de handicap ou en situation de participation sociale.

Le concept de résilience permet de mieux évaluer ou de mieux nommer le résultat de l'interaction entre les facteurs de risque et les facteurs de protection. Il permet essentiellement de créer un continuum «situation de vulnérabilité et situation de résilience», qui nous aide à mieux décrire les contextes de vie de la personne. Il s'agit de faire en sorte que ces personnes grandement «vulnérables» deviennent, dans la mesure du possible, des personnes qui agissent à partir de comportements résilients.

Il nous faut tenir compte du fait que la signification qu'une personne accorde à un événement qu'elle a vécu, qu'il soit traumatisant ou non, dépend du degré d'organisation de ses structures cognitives, de l'influence de l'intelligence émotionnelle sur la perception accordée à l'événement et du type de confirmation apporté par sa famille ou son entourage face au sens à donner à cet événement.

Des difficultés supplémentaires s'ajoutent quand on considère le processus de résilience chez des personnes dont la naissance même est liée à une situation traumatique. Le développement des capacités propres à l'émergence du processus de résilience est grandement déficitaire ou dysharmonieux de sorte que la personne ne peut compter sur ses propres ressources pour assurer son adaptation aux diverses situations de vie avec lesquelles elle doit composer. Il en est ainsi des personnes qui souffrent d'un syndrome génétique particulier qui se manifeste par de nombreuses déficiences ou troubles associés. La résilience personnelle, qui est tout de même possible, est grandement tributaire des effets de la résilience familiale et de la disponibilité des ressources de l'environnement physique et social pouvant assurer un soutien technique et humain auprès des familles. Dans ce contexte comme dans bien d'autres, nous constatons l'impact prépondérant des facteurs environnementaux sur les facteurs génétiques.

Le fait d'opposer la notion de résilience à celle de vulnérabilité vient compléter notre orientation qui consiste à rendre positives nos interventions en la centrant sur le développement des capacités de la personne plutôt que sur la diminution des facteurs limitatifs. S'il est nécessaire de bien saisir l'influence des facteurs de risque, c'est sur le développement des facteurs de protection que doivent s'établir les bases de l'accompagnement. Réfléchir en termes de mise en place des facteurs de protection permet, comme nous l'avons précisé, de mieux impliquer les personnes et les familles dans la planification de services. Contribuer à la réalisation d'un projet situe mieux le sens de nos actions que le fait de répondre à un ensemble de besoins identifiés par les personnes et leur famille. S'associer à un projet véhicule plus d'espoir que le fait de chercher à répondre à des besoins, ce qui

\section{S'IL FAUT DEUX ÉLÉMENTS POUR}

FAIRE UN TRAUMATISME, L'UN

DANS LE RÉEL ET L'AUTRE DANS

LA REPRÉSENTATION DU RÉEL,

IL FAUT AUSSI DEUX ÉLÉMENTS

POUR QUE SE DÉVELOPPENT

DES ATTITUDES DE RÉSILIENCE :

UNE RÉALISATION PERSONNELLE

ET UNE APPRÉCIATION DE

CETTE RÉALISATION PAR AUTRUI.

nous place immanquablement devant nos limites et celles du réseau de services. Dans le premier cas, les principes d'égalité et de respect priment sur les notions d'inégalité et de dépendance. La résilience nous amène à «penser et agir autrement», en délaissant les expressions de «personnes à risque » ou «familles à risque» pour nous centrer sur l'émergence de comportements autonomes et résilients.

S'il faut deux éléments pour faire un traumatisme, l'un dans le réel et l'autre dans la représentation du réel, il faut aussi deux éléments pour que se développent des attitudes de résilience: une réalisation personnelle et une appréciation de cette réalisation par autrui. Comme le soulignent plusieurs auteurs, la résilience passe nécessairement par le regard appréciatif de l'autre sur soi.

\section{LA RÉSILIENCE EN CONTEXTE}

Le but de notre texte était de faire ressortir la place centrale que peut occuper le concept de résilience dans la façon d'accompagner au quotidien les personnes qui présentent des incapacités intellectuelles, leur famille et les intervenants qui sont impliqués dans leur situation. Nous avons établi certains liens entre la résilience en tant que processus et la philosophie d'accompagnement des personnes en situant les actions des intervenants non plus sur la modification de certaines caractéristiques des personnes mais sur l'ensemble de son contexte de vie. Nous avons tissé certains liens entre les notions de résilience et de déficience intellectuelle en dépassant le sens de «capacité » habituellement accordé au terme résilience pour migrer vers celui de «processus ${ }^{5}$ qui met en relief le rôle de soutien accordé par les familles et les divers intervenants du réseau de services. Un survol du processus de production nous a permis de situer le rôle de divers facteurs dans le fait qu'une personne se trouve ou non en situation de handicap à cause de la prédominance des facteurs de risque ou de l'impact des facteurs de protection sur ces derniers.

Il s'agit en fait d'une première articulation entre ces concepts qui permettra, nous le souhaitons, de considérer la personne qui présente des incapacités intellectuelles comme pouvant développer certaines aptitudes qui lui permettront de réaliser diverses activités ou d'exercer certains rôles sociaux si elle reçoit de la part de leur famille et du réseau de services, le soutien correspondant à leur situation personnelle.

\section{Bibliographie}

AMERICAN ASSOCIATION ON MENTAL RETARDATION (2002). Definition, Classification, and Systems of Supports, $10^{\mathrm{e}}$ édition, Washington, D.C.

ANAUT, M. (2007). "Humour et résilience à l'école», dans B. CYRULNIK et J.P. POURTOIS (dir.), École et résilience, Paris, Odile Jacob, p. 327-350.

ANAUT, M. (2006). "La résilience au risque de la psychanalyse ou la psychanalyse au risque de la résilience? », dans B. CYRULNIK et P. DUVAL (dir.), Psychanalyse et résilience, Paris, Odile Jacob, p. $77-104$

BALEGNO, L. et M.E. COLMANERES (2003). «Les enfants des rues en Colombie», dans FONDATION POUR L'ENFANCE, M.P. POILPOT (dir.), La résilience, Le réalisme de l'espérance, Ramonville-SaintAgne, Éditions Érès, p. 156.

BERNARDINI, S.C. et J.M. JENKINS (2002). Vue d'ensemble des risques et des facteurs de protection pour les enfants touchés par la séparation et le divorce. Institut d'études pédagogiques de l'Ontario, Ministère de la Justice du Canada, 71 p. 
BOISVERT, Y. (2006). "Modèle clinique d'application du cadre conceptuel du PPH auprès des personnes présentant des incapacités intellectuelles », dans H. GASCON, D. BOISVERT, M.C. HAELEWYCK et al. (dir.), Déficience intellectuelle: savoirs et perspectives d'action, tome 1, Représentations, diversité, partenariat et qualité, Québec, Presses Inter Universitaires, p. 31-42.

BOISVERT, Y. (à paraître automne 2010). Fondements théoriques de l'accompagnement et de l'intervention spécialisés en contexte auprès des personnes présentant des incapacités intellectuelles, Cap-Rouge, Québec, Presses Inter Universitaires, 98 p.

BOUVIER, P. (2001). "Tempérament, risque et résilience chez l'enfant», dans M. MANCIAUX (dir.), La résilience: résister et se construire, Genève, Éditions Médecine et Hygiène, p. 47-55.

CYRULNIK, B. (2001). «Neuro-éthologie de la résilience", dans M. MANCIAUX (dir.), La résilience: résister et se construire, Genève, Éditions Médecine et Hygiène, p. 137.

DAMASIO, A. (2003). Spinoza avait raison, Joie et tristesse, le cerveau des émotions, Paris, Odile Jacob, 346 p.

FOUGEYROLlAS, P., R. CLOUTIER, H. BERGERON et J. CÔTÉ (1998). Classification québécoise, Processus de production du handicap, Lac-St-Charles (Québec), Réseau international sur le processus de production du handicap, $163 \mathrm{p}$.

GARMEZY, N. (1993). "Children in poverty: Resilience despite risk», Psychiatry Interpersonal and Biological Processes, vol. $56, \mathrm{n}^{\circ} 1$, p. 127-136.

LECOMTE, J. (2004). Guérir de son enfance, Paris, Odile Jacob, 382 p.
MANCIAUX, M., S. VANISTENDAEL, J. LECOMTE et B. CYRULNIK (2001). "La résilience: état des lieux», dans $\mathrm{M}$. MANCIAUX (dir.), La résilience, Résister et se construire, Genève, Cahiers médicosociaux, p. 17.

MICHAUD, P.A. (2003). "Une approche clinique fondée sur la résilience», dans FONDATION POUR L'ENFANCE, M.P. POILPOT (dir.), La résilience, Le réalisme de l'espérance, Ramonville-Saint-Agne, Érès, p. 280.

MISÈS, R., R. PERRON et R. SALBREUX (1994). Retards et troubles de l'intelligence de l'enfant, Paris, ESF Éditeur, coll. «La vie de l'enfant», $301 \mathrm{p}$.

PETIT, M., M. LALOU-MOATTI et P. CLERVOY (1999). "Santé mentale, risque, vulnérabilité, ressources", dans S. LEBOVICI (dir.) (1999), Nouveau traité de psychiatrie de l'enfant et de l'adolescent, tome 4, Paris, Quadrige, p. 3041-3046.

PLOMIN, R., J.C. DEFRIES, G.MC. CLEARN et al. (1999). "Des gènes au comportement", dans M. MANCIAUX (dir.) (2001), La résilience, Résister et se construire, Genève, Éditions Médecine et Hygiène, p. 137.

RUTTER, M. et M. RUTTER. (1993). Developing Minds: Challenge and Continuity Across the Life Span, New York, Basic Books, $418 \mathrm{p}$.

SCHALOCK, R.L., R.A. LUCKASSON et K.A. SHOGREN (2007). "The renaming of mental retardation: Understanding the change to the term intellectual disability ", Intellectual and Developmental Disabilities, vol. 45, $\mathrm{n}^{\circ} 2$, p. 116-124.

TERRISSE, B., J.C. KALUBI et S.J. LARIVÉE (2007). "Résilience et handicap chez l'enfant», Reliance, vol. 2, n 24, p. $12-21$.
TERRISSE, B. et M.L. LEFEBVRE (2007). «L'école résiliente: facteurs de risque, facteurs de protection et sentiment d'efficacité collective», dans B. CYRULNIK et J.P. POURTOIS (dir.), École et résilience, Paris, Odile Jacob, p. 47-83.

THEIS, A. (2001). «La résilience dans la littérature scientifique», dans M. MANCIAUX (dir.), La résilience, Résister et se construire, Genève, Cahiers médico-sociaux, p. 34.

TREMBLAY, M. (1992). L'adaptation humaine, Un processus biopsychosocial à découvrir, Montréal, Éditions Saint-Martin, $400 \mathrm{p}$.

VANISTENDAEL, S. (1998). "Clés pour devenir: la résilience», dans B. CYRULNIK (1999), Un merveilleux malheur, Paris, Odile Jacob, 218 p.

WEHMEYER, M.L., W.H.E. BUNTINX, Y. LACHAPELLE et al. (2008). "The intellectual disability construct and its relation to human functioning", Intellectual and Developmental Disabilities, vol. 46, $\mathrm{n}^{\circ} 4$, p. 311-318.

\section{Notes}

1. Ces mouvements sociaux sont définis dans la plupart des ouvrages concernant la déficience intellectuelle dont celui à paraître intitulé Fondements théoriques de l'accompagnement et de l'intervention spécialisés en contexte auprès des personnes présentant des incapacités intellectuelles écrit par Yves Boisvert.

2. Nous pourrions, sans craindre d'être contredit, affirmer que dans le PPH les aspects positifs du modèle correspondent aux facteurs de protection et que, dans une prochaine version du modèle, les notions de résilience et de facteurs de protection seront formellement intégrées. 